\title{
Vasopressin in septic shock: pressing questions remain
}

\author{
Robert Keyes, MD · Peter G. Brindley, MD
}

Received: 10 September 2008/Revised: 16 October 2008/Accepted: 27 October 2008/Published online: 19 December 2008

(c) Canadian Anesthesiologists' Society 2008

\section{Article appraised}

Russell JA, Walley KR, Singer J, et al.; VASST Investigators. Vasopressin versus norepinephrine infusion in patients with septic shock. N Engl J Med 2008; 258: 877-87.

\section{Structured Abstract}

Background: The use of low-dose vasopressin as an adjunctive vasopressor in septic shock is common, but its effect on mortality is unknown.

Objective: To determine whether a low-dose vasopressin infusion reduces mortality in septic shock when added to an infusion of norepinephrine.

Design and Setting: Multicentre, multiple-blinded, randomized controlled trial conducted in three countries from July, 2001 to April, 2006.

Patients: Inclusion criteria were patients $\geq 17$ years of age with septic shock requiring $\geq 5$ ug $\mathrm{min}^{-1}$ of norepinephrine (or equivalent) for $\geq 6 \mathrm{~h}$, or requiring $\geq 15 \mathrm{ug} \mathrm{min}^{-1}$ of norepinephrine (or equivalent) for $\geq 3 \mathrm{~h}$, despite having received $\geq 500 \mathrm{ml}$ of normal saline. Study enrolment was only permitted within $24 \mathrm{~h}$ of meeting inclusion criteria. Exclusion criteria included previous use of vasopressin, acute myocardial infarction, New York Heart Association class III or IV congestive heart failure, acute mesenteric ischemia, severe traumatic brain injury, hyponatremia $<130$ mmol $^{-1}$, Raynaud's phenomenon, systemic sclerosis, vasospastic diathesis, pregnancy, an irreversible condition with an estimated six-month mortality $\geq 50 \%$,

R. Keyes, MD $(\varangle) \cdot$ P. G. Brindley, MD University of Alberta, Edmonton, Canada e-mail: rkeyes@ualberta.ca anticipated death within $12 \mathrm{hr}$, or the decision not to proceed with aggressive care.

Intervention: Study patients were randomized to a blinded infusion of vasopressin (started at $0.01 \mathrm{U} \mathrm{min}^{-1}$ and titrated to $0.03 \mathrm{U} \mathrm{min}^{-1}$ over $40 \mathrm{~min}$ ) or norepinephrine (started

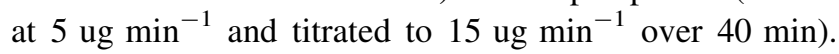
Additional open-label vasopressors (excluding vasopressin) were administered as necessary to maintain a target mean arterial pressure (MAP) of $65-75 \mathrm{mmHg}$. The study drug could be tapered after the target MAP had been maintained for $>8 \mathrm{~h}$ without open-label vasopressors.

Measurements: The primary outcome was 28-day mortality. Secondary outcomes included 90-day mortality, rates of adverse events, and markers of clinical illness (e.g., organ dysfunction scores, need for vasopressors, mechanical ventilation, and renal replacement therapy). A priori subgroup analysis was performed on patients with less severe $v s$ more severe septic shock (defined by the need for $5-14$ ug $\min ^{-1}$ vs. $\geq 15$ ug $\min ^{-1}$ of norepinephrine, respectively, at the time of study enrolment).

Main results: Seven hundred seventy-eight patients were included in the final analysis. There were no statistically significant differences between the vasopressin and the norepinephrine groups in terms of 28-day mortality (35.4 vs. $39.3 \%$, respectively, $P=0.26$ ), 90-day mortality (43.9 vs. $49.6 \%$, respectively, $P=0.11$ ), rates of organ dysfunction, or overall rates of adverse events. Patients with less severe septic shock who were randomized to vasopressin had a trend towards decreased 28-day mortality ( 26.5 vs. $35.7 \%$, respectively, $P=0.05$ ) and 90 -day mortality (35.8 vs. $46.1 \%$, respectively, $P=0.04$ ).

Conclusions: An adjunctive vasopressin infusion does not reduce overall mortality in septic shock, but may benefit patients with less severe septic shock. 


\section{Commentary}

\section{Study relevance}

Sepsis is the leading cause of death in non-coronary intensive care units (ICUs), with $>200,000$ deaths annually in the United States and cost, incidence, and mortality comparable to acute coronary syndromes. ${ }^{1,2}$ Despite this fact, the optimal agent to augment blood pressure in septic shock remains unclear. Norepinephrine is typically a firstline agent, but the discovery of endogenous vasopressin deficiency in septic shock $^{1,3,4}$ has fostered the use of exogenous vasopressin as an adjunct. Nonetheless, mortality data remains limited. While two small randomized controlled trials (RCTs) have previously examined the role of vasopressin in septic shock, ${ }^{5,6}$ neither study was adequately powered to evaluate mortality, organ dysfunction, or safety. ${ }^{2}$ As such, the "Vasopressin and Septic Shock Trial” (VASST) by Russell et al. was eagerly anticipated.

\section{Analysis of methodology}

Russell et al. deserve credit for a well-conducted, multicentre RCT that screened 6,229 patients in three countries (i.e., Canada, Australia, and the United States) over five years. However, only 802 patients $(12.9 \%)$ were ultimately enrolled, and only 778 patients $(12.5 \%)$ were included in the final analysis. Furthermore, while the investigators predicted that 776 patients would be needed to achieve $80 \%$ power to detect a $10 \%$ mortality difference, this calculation assumed a $60 \%$ mortality rate in the norepinephrine (i.e., control) group. Unfortunately, since the actual mortality rate was $<40 \%$, this study was underpowered.

Concealment of allocation and randomization procedures were appropriate to the study design, with the investigators using central telephone access and a computer-generated, stratified randomization list. Blinding was similarly meticulous, with only the study pharmacists aware of the treatment assignments for the duration of the study (as well as during the final data analysis). The experimental and control groups were well matched at baseline. The average age was approximately 60 years in both groups, and the average Acute Physiology and Chronic Health Evaluation (APACHE) II score in both groups was 27. Regarding the open-label catecholamines being used at the time of enrolment, approximately $85 \%$ of patients in both groups were receiving norepinephrine (with an average infusion rate of $20.7 \mathrm{ug} \mathrm{min}^{-1}$ ), and approximately $30 \%$ of patients in both groups were receiving infusions of multiple agents. Also, the sources of infection were similar between the two groups and were reflective of the distribution seen in typical practice (i.e., approximately $40 \%$ pulmonary, $25 \%$ abdominal, and $30 \%$ "other" sites). Unfortunately, the paper did not outline either the amount of fluid administered prior to study enrolment or the administration of adequate antibiotics. The authors state (correctly) that the effect of co-interventions was minimized by treatment concealment. However, prompt fluids and broad-spectrum antibiotics may be the greatest determinant of outcome in septic shock. $^{7-9}$ Knowing that the two groups were matched in this regard would help clinicians to better assess the effect of vasopressin in the context of timely and comprehensive care.

\section{Application to clinical practice}

Overall, there were no statistically significant differences in 28-day mortality, 90-day mortality, organ dysfunction, or serious adverse events.

Of note, the authors predicted that vasopressin would be more efficacious in more severe septic shock. However, this subgroup showed no significant difference in mortality with vasopressin as compared to norepinephrine (28-day mortality $44.0 \%$ vs $42.5 \%$, respectively, $P=0.76$; 90-day mortality $51.8 \%$ vs $52.8 \%$, respectively, $P=0.84$ ). In contrast, patients with less severe septic shock had a trend towards both decreased 28-day mortality (26.5\% vs. $35.7 \%$, respectively, $P=0.05$ ) and decreased 90-day mortality (35.8\% vs. $46.1 \%$, respectively, $P=0.04$ ) with vasopressin. However, despite the fact that these less severely affected patients were an a priori defined subgroup, this was only a secondary outcome. As such, the authors correctly concluded that this finding should be regarded as hypothesis-generating only. ${ }^{2}$

Like many RCTs, VASST had numerous exclusion criteria that could potentially limit its external validity. Notably, patients with acute ischemic heart disease or heart failure were excluded. Without these exclusions, vasopressin may potentially have been associated with an increased mortality rate. ${ }^{2}$ In addition, the requirement for only $500 \mathrm{ml}$ of normal saline prior to enrolment was lower than the requirement in typical practice. In comparison, Rivers' study ${ }^{7}$ on early goal-directed therapy used crystalloid challenges of $20-30 \mathrm{ml} \mathrm{kg}^{-1}$ over $30-\mathrm{min}$. Similarly, the 2008 Surviving Sepsis Campaign guidelines recommend multiple crystalloid boluses of $1000 \mathrm{ml}$ for sepsis-related tissue hypoperfusion. ${ }^{8}$ Treatment in VASST was also initiated an average of $12 \mathrm{~h}$ after the onset of septic shock. In contrast, work by Rivers et al. ${ }^{7}$ and Kumar et al. ${ }^{9}$ suggests that hemodynamic optimization and broad spectrum antibiotics offer the greatest survival benefit when administered within one to $2 \mathrm{~h}$. As such, the patients in VASST may simply have been treated too late to demonstrate a mortality effect. 
Regarding serious adverse events, eight of ten cases of digital ischemia occurred in the vasopressin group, with one patient in this group requiring surgical intervention. In contrast, eight of 11 cardiac arrests occurred in the norepinephrine group-despite no difference between the two groups in regards to ischemia or acute myocardial infarction rates. Additional secondary outcomes included ICU and hospital lengths-of-stay, as well as the number of days free from organ dysfunction, vasopressors, mechanical ventilation, and renal replacement therapy. While these outcomes were not analyzed using intention-to-treat analysis, none demonstrated a statistically significant difference.

\section{Clinical perspective}

Since the average MAP at the time of enrolment in the VASST study was already $>70 \mathrm{mmHg}$, Russell et al. did not actually examine the role of vasopressin in "catecholamine-refractory" septic shock. (In this setting, the potential benefit of vasopressin remains inadequately studied). ${ }^{2}$ Instead, VASST examined the role of vasopressin as a "catecholamine-sparing" agent.

While low-dose vasopressin infusions appear to be safe in septic shock, based on the VASST study, a mortality benefit cannot be strongly recommended. ${ }^{2}$ First, the extensive exclusion criteria in this study complicate its application to clinical practice. Secondly, the failure to outline fluid or antibiotic administration prior to enrolment makes it difficult to verify baseline equivalence between the two study groups. Finally (and perhaps most importantly), an adequately powered validation study is first needed to both confirm the potentially encouraging results seen in less severe shock and to rule out a significant trend towards harm in more severe shock. While the overall body of evidence supports early hemodynamic optimization in septic shock, ${ }^{7,9}$ VASST suggests that an optimal vasopressor has not yet been identified. Ultimately, when a patient is treated may end up being equally as important as how a patient is treated. At present, many "pressing" questions remain.

Funding sources None.

Conflicts of interest None declared.

\section{References}

1. Angus D, Linde-Zwirble WT, Lidicker J, Clermond G, Carcillo J, Pinsky MR. Epidemiology of severe sepsis in the United States: analysis of incidence, outcome, and associated costs of care. Crit Care Med. 2001; 29: 1303-10.

2. Parrillo JE. Septic shock-vasopressin, norepinephrine, and urgency. N Engl J Med. 2008; 358: 954-6.

3. Landry $D W$, Oliver JA. The pathogenesis of vasodilatory shock. $\mathrm{N}$ Engl J Med. 2001; 345: 588-95.

4. Holmes CL, Patel BM, Russell JA, Walley KR. Physiology of vasopressin relevant to management of septic shock. Chest. 2001; 120: 989-1002.

5. Patel BM, Chittock DR, Russell JA, Walley KR. Beneficial effects of short-term vasopressin infusion during severe septic shock. Anesthesiology. 2002; 96: 576-82.

6. Malay MB, Ashton RC Jr, Landry DW, Townsend RN. Low-dose vasopressin in the treatment of vasodilatory septic shock. J Trauma. 1999; 47: 699-703.

7. Rivers E, Nguyen B, Havstad S. Early Goal-Directed Therapy Collaborative Group. Early goal-directed therapy in the treatment of severe sepsis and septic shock. N Engl J Med. 2001; 345: 136877.

8. Dellinger RP, Levy MM, Carlet JM, et al. Surviving Sepsis Campaign: International guidelines for management of severe sepsis and septic shock: 2008. Crit Care Med. 2008; 36: 296-327.

9. Kumar A, Roberts D, Wood KE, et al. Duration of hypotension before initiation of effective antimicrobial therapy is the critical determinant of survival in human septic shock. Crit Care Med. 2006; 34: 1589-96. 